\title{
Prevalence and Determinants of Home Delivery in Eastern Africa: Further Analysis of Countries Recent Demographic and Health Survey Data
}

\section{Lemma Demissie Regassa}

Haramaya University

Assefa Tola

Haramaya University

Adisu Birhanu Weldesenbet

Haramaya University

Biruk Shalmeno Tusa ( $\sim$ Birukshalmeno27@gmail.com )

Haramaya University

\section{Research Article}

Keywords: Home delivery, Eastern Africa, Demographic and Health Survey Data

Posted Date: February 25th, 2021

DOI: https://doi.org/10.21203/rs.3.rs-239066/v1

License: (c) (i) This work is licensed under a Creative Commons Attribution 4.0 International License. Read Full License 


\section{Abstract}

Background: Despite the high proportion of maternal mortality ratio in East African countries primarily attributed to home delivery, overall magnitude of home delivery and its determinants remains unclear. Therefore, the current study aimed to determine magnitude of home delivery and its determinant factors in East Africa using Demographic and Health Survey (DHS) data.

Methods: We pooled the DHS survey data of the 11 East African countries, and a total weighted sample of 125,786 women were included in the study. Generalized Linear Mixed Models (GLMM) was fitted to identify factors associated with home delivery. Variables with Adjusted Odds Ratio (AOR) with a 95\% Confidence Interval $(\mathrm{Cl})$, and $p$-value $<0.05$ in the final GLMM model were reported to declare significantly associated factors with home delivery.

Result: The weighted prevalence of home delivery was $23.79 \%$ [95\% Cl: 23.55 - 24.02] among women in East Africa countries. Home delivery was highest among Ethiopian women (72.5\%) whereas, it was lowest among women from Mozambique (2.8\%). In GMM, respondent's age group, marital status, educational status, place of residence, living country, wealth index, media exposure and total children ever born were shown significantly associated with the home delivery in the East Africa counties

Conclusion: Home delivery was varied between countries of East African zone. The home delivery was significantly increased among women aged 20-34 years, higher number of ever born children, rural residence, never married or formerly married participants. To the contrast home delivery was decreased with higher educational level, media exposure, and higher wealth index. Wide range interventions to decrease home delivery should focus on addressing inequities associated with maternal education, family wealth, increased access to media, as well as narrowing the gap between the rural and the urban areas, poor and rich families, and married and unmarried mothers.

\section{Background}

Maternal mortality remains a major public health problem globally. Sub-Saharan African (SSA) regions bear the highest burden with $85 \%$ of maternal deaths reported from the region. Studies indicated every year 529,000 maternal deaths and four million newborn deaths in the first week of life occur worldwide [1, 2]. The estimated maternal mortality ratio (MMR) in developing countries ( 239 per 100,000 live births) is 20 times higher compared to the developed regions (12 maternal deaths per 100,000 live births). Despite the great improvement over the last decades, the drop in maternal mortality is far from reaching a target decline of reaching less than 70 MMR by 2030 at the current pace $[3,4]$.

Most of maternal deaths in sub-Saharan Africa are highly attributed to home delivery with most births occurring at home. In low and middle-income countries (LMIC), many deliveries still occur at home without the assistance of trained attendants [2, 5]. Mothers deliver in an unhygienic environment, without a skilled birth attendant and lifesaving medications. Sub-Saharan Africa and South Asia together contribute over $85 \%$ of maternal deaths, and of which only half of deliveries are at home $[6,7]$. The 
negative impact of home delivery extends to child and responsible for neonatal morbidity and mortality. Since home deliveries are attended by unskilled health professionals and occurs in an unsafe environment, they leads to adverse neonatal and maternal outcomes like the increased risk of infection, postpartum hemorrhage (PPH), and transmission of HIV/AIDS to relatives or traditional birth attendants, who conduct deliveries without protective equipment. Most of these maternal deaths are preventable if timely and appropriate interventions are applied $[8,9]$.

Evidences showed that even though skilled birth attendance can save the lives of women, only $59 \%$ of births were attended by skilled birth attendants between 2012 and 2017 in sub-Saharan Africa. High load of home delivery in the region is a precipitating factor for the high maternal mortality ratio. The large proportion of direct cause of maternal death including obstetric complications such as hemorrhage, pregnancy-induced hypertension, sepsis, and obstructed labor which collectively accounts for $64 \%$ of maternal deaths could be prevented primarily by making the delivery attended by a skilled birth attendant at a health facility $[10,11]$.

Despite the high proportion of maternal mortality ratio in East African countries primarily attributed to home delivery, overall magnitude of home delivery and its determinants remains unclear. Therefore, the current study aimed to determine magnitude of home delivery and its determinant factors in East Africa using Demographic and Health Survey (DHS) data. The finding of the current study provides evidence for health planners, decision makers, stakeholders, and health professionals in planning for further reduction of home delivery which is helpful in turn to decrease maternal mortality in low-income and middle-income countries.

\section{Methods}

\section{Study setting, design, and period}

We conducted Pooled analysis based on Demographic and Health Surveys (DHS) conducted in the 11 East African countries (including Burundi, Comoros, Ethiopia, Kenya, Malawi, Mozambique, Rwanda, Tanzania, Uganda, Zambia and Zimbabwe) from 2012 to 2017. The DHS is considered as the main data source as it was designed to provide population and health indicators at the national and regional levels. The data collection period was varying but includes the data of five years prior to the survey. This further data analysis was conducted from January to February, 2021.

Based on Updated country income classifications for the World Bank's 2020 fiscal year Burundi, Ethiopia, Malawi, Mozambique, Rwanda, Tanzania and Uganda are low income countries while Comoros, Kenya, Zambia and Zimbabwe are low middle income countries [12].

\section{Data source and sampling}

Data was obtained from the measure DHS program at www.measuredhs.com website after we submitted concept notes about the project. We pooled the most recent DHS data from the 11 East Africa Countries. 
There are 20 countries in World Health Organization (WHO) regions of East Africa. In history, only 13 of these countries had DHS data. For this study, 11 countries were included [13] (Fig. 1).

The DHS used two stages of stratified sampling technique to select the study participants. In the first stage, Enumeration Areas (EAs) were randomly selected. In the second stage households were selected. We pooled the DHS survey data of the 11 East African countries, and a total weighted sample of 125,786 women who had history of bearing child in the last 5 years preceding the survey day was included in the study.

\section{Data collection methods}

The DHS program adopts standardized methods involving uniform questionnaires, manuals, and field procedures to gather the information that is comparable across countries in the world. It is the representative household surveys that capture data from a wide range of monitoring and impact evaluation indicators in the area of population, health, and nutrition with face to face interviews of women age 15 to 49. Each country's survey consists of different datasets including men, women, children, birth, and household datasets. Detailed survey methodology and sampling methods used in gathering the data have been reported elsewhere [14]. For this study, we used the Individual record dataset (IR file) contained information of eligible women aged 15 to 49 years in each country.

\section{Variables and measurement}

\section{Outcome variable}

The outcome variable of this study was a home delivery. The response variable was generated from the question asked to the women who gave birth within 5 years preceding the survey question. The response was dichotomized as a home delivery and institutional delivery (if delivered at any type of health institutions). Home delivery includes the option given in the survey question termed as respondents home, and others home. Health institutions include government hospitals, health centers, health posts, private clinics or private hospitals. If women delivery at home, we coded as " 1 ", otherwise coded as " 0 ".

\section{Independent variables}

Country, age, marital status, educational level, place of residence, wealth index, sex of head of household, age of head of household, media exposure, total children ever born were included as independent variables in this study

\section{Statistical analysis}

The variables were extracted using the IR file. We use STATA software version 16.0 to clean, recode, and analysis the pooled data. After joining the extracted data from the 11 East African countries, we weighted data using women's individual sample weight (v005), and strata (v021). The proportion of home delivery was described and presented using pie chart. The DHS data had a hierarchical structure as women were nested within a cluster, and clusters with in the country. Hence the data violates the independency of the 
observation as the women may share similar characteristics within the cluster (and /or country). This implies that there is a need to consider the between cluster variability by using Generalized Linear Mixed Models. Likelihood Ratio test (LR), Intra-cluster Correlation Coefficient (ICC), Median Odds Ratio (MOR), and Proportional Change in Variance (PCV) were computed to measure the variation between clusters. The ICC quantifies the proportion of the total observed difference in home delivery attributable to cluster variations (degree of heterogeneity). On the other hand, MOR was used to quantify the variation or heterogeneity in home delivery between clusters. Hence, MOR is defined as the median value of the odds ratio between the cluster high odds of home delivery and cluster at lower odds of home delivery when randomly picking out two clusters /EAs. Finally, PCV measures the total variation of home delivery attributed to individual-level and community-level factors in the final model compared to the null model. The detail description and formulas for ICC [15], MOR [16] and PCV [16] are described elsewhere. The null model, individual level, clusters level and both cluster and individual level factors were fitted. Model comparison was made based on deviance (- 2LLR) since the models were nested. Finally, model with both at individual and cluster level factors were selected.

Variables with $p$-value $\leq 0.2$ in the bi-variable analysis for both individual and community-level factors were fitted in the multivariable model. Variables with Adjusted Odds Ratio (AOR) with a 95\% Confidence Interval $(\mathrm{Cl})$, and $p$-value $<0.05$ in the final GLMM model were reported to declare significantly associated factors with home delivery.

\section{Results}

\section{Socio-demographic characteristics}

In this study, a total of 125,696 women who gave birth in the 5 years preceding each country's DHS survey were included. Majority (26.93\%) of women were in age group $25-29$ years followed $(24.05 \%)$ by 20-24 years. The median age of women was 28.3 with IQR of 23.5-33.9 years. Currently married women accounted for the large majority (85.35\%) of the study participants. Half (52.99\%) of women were attend primary education. More than three- fourth $(77.22 \%)$ of the study participants were living in rural residents. Similarly, males were the head of the household in three out four $(76.42 \%)$ of the study participants.

Country with the highest proportion of study participants was Kenya $(15.48 \%)$ followed by Malawi (13.82\%) and Uganda (12.14\%). Whereas Comoros (2.54\%) and Zimbabwe (5.10\%) were countries with the smallest number of women included in the study. About two third $(65.41 \%)$ of the participants reported exposure to media. The total number of children ever born from the women was ranging from 1 to 18 with mean ( \pm SD) of 3.81 and 2.38. Majority (54.46\%) of women gave $1-3$ children in their life time (Table 1). 
Table 1

Background characteristics of women in East Africa countries, 2021.

\begin{tabular}{|c|c|c|}
\hline Variables & Weighted frequency & Percent \\
\hline \multicolumn{3}{|l|}{ Age } \\
\hline $15-19$ & 7,090 & 5.64 \\
\hline $20-24$ & 30,256 & 24.05 \\
\hline $25-29$ & 33,880 & 26.93 \\
\hline $30-34$ & 26,520 & 21.08 \\
\hline $35-39$ & 17,685 & 14.06 \\
\hline $40-44$ & 8,023 & 6.38 \\
\hline $45-49$ & 2,332 & 1.85 \\
\hline \multicolumn{3}{|l|}{ Marital status } \\
\hline Never married & 6,140 & 4.88 \\
\hline Currently married & 107,363 & 85.35 \\
\hline Formerly/ever married & 12,283 & 9.76 \\
\hline \multicolumn{3}{|l|}{ Educational level } \\
\hline Uneducated & 30,095 & 23.93 \\
\hline Primary & 66,653 & 52.99 \\
\hline Secondary & 24,513 & 19.49 \\
\hline Higher & 4,515 & 3.59 \\
\hline Don't know & 10 & 0.01 \\
\hline \multicolumn{3}{|l|}{ Place of residence } \\
\hline Urban & 28,654 & 22.78 \\
\hline Rural & 97,132 & 77.22 \\
\hline \multicolumn{3}{|l|}{ Country } \\
\hline Burundi & 13,611 & 10.82 \\
\hline Comoros & 3,198 & 2.54 \\
\hline Ethiopia & 11,023 & 8.76 \\
\hline Kenya & 19,474 & 15.48 \\
\hline
\end{tabular}




\begin{tabular}{|c|c|c|}
\hline Variables & Weighted frequency & Percent \\
\hline Malawi & 17,384 & 13.82 \\
\hline Mozambique & 11,512 & 9.15 \\
\hline Rwanda & 8,002 & 6.36 \\
\hline Tanzania & 10,052 & 7.99 \\
\hline Uganda & 15,270 & 12.14 \\
\hline Zambia & 9,841 & 7.82 \\
\hline Zimbabwe & 6,419 & 5.10 \\
\hline \multicolumn{3}{|l|}{ Wealth index } \\
\hline Poorest & 29,852 & 23.73 \\
\hline Poorer & 26,977 & 21.45 \\
\hline Middle & 24,199 & 19.24 \\
\hline Richer & 23,283 & 18.51 \\
\hline Richest & 21,475 & 17.07 \\
\hline \multicolumn{3}{|c|}{ Sex of head of household } \\
\hline Male & 96,121 & 76.42 \\
\hline Female & 29,665 & 23.58 \\
\hline \multicolumn{3}{|c|}{ Age of head of household } \\
\hline $15-19$ & 643 & 0.51 \\
\hline $20-24$ & 9,217 & 7.33 \\
\hline $25-29$ & 22,860 & 18.17 \\
\hline $30-34$ & 26,294 & 20.90 \\
\hline $35-39$ & 22,717 & 18.06 \\
\hline $40-44$ & 15,234 & 12.11 \\
\hline $45-49$ & 9,477 & 7.53 \\
\hline$>49$ & 19,3445 & 15.38 \\
\hline \multicolumn{3}{|c|}{ Media exposure } \\
\hline No & 43,481 & 23.58 \\
\hline Yes & 82,230 & 65.41 \\
\hline
\end{tabular}




\begin{tabular}{|lll|}
\hline Variables & Weighted frequency & Percent \\
\hline Total children ever born & & \\
\hline $1-3$ & 68,4967 & 54.46 \\
$4-6$ & 39,571 & 31.46 \\
\hline $7-9$ & 14,698 & 11.68 \\
$>9$ & 3,020 & 2.40 \\
\hline
\end{tabular}

\section{Prevalence of home delivery}

The weighted prevalence of home delivery was 23.79\% [95\% Cl: 23.55-24.02] among women in East Africa countries (Fig. 2). Home delivery was highest among Ethiopian women (72.5\%), followed by Kenyan women (37.5\%) and Tanzanian women (34.7\%). On the other hand, it was lowest among women from Mozambique (2.8\%), Malawi (7.1\%) and Rwanda (7.9\%).

Home delivery was higher (34.0\%) among women age group 45-49 years followed $40-44$ years (30.5\%) and $35-39$ years $(27.5 \%)$. Similarly, home delivery was higher among currently married women (24.5\%), uneducated women (39.6\%), women from rural area (27.9\%), and women with lower economic status (36.2\%). The proportion of home delivery was higher among women living in household head by male (24.5\%), women who have no media exposure (32.5\%), and women who ever born greater than nine children (42.7\%). The prevalence of home delivery was progressively increased from $14 \%$ among women from households headed by person aged $14-19$ years to $27.5 \%$ among the head with age group $40-44$ years and then slightly decreased at each age group (Table 2). 
Table 2

Shows distribution of home delivery among women in east Africa countries, 2021.

\begin{tabular}{|c|c|c|c|}
\hline \multirow[t]{2}{*}{ Variables } & \multicolumn{2}{|l|}{ Home delivery } & \multirow[t]{2}{*}{ Total } \\
\hline & Yes (\%) & No (\%) & \\
\hline \multicolumn{4}{|l|}{ Country } \\
\hline Burundi & $1,621(11.9)$ & $11,990(88.1)$ & 13,611 \\
\hline Comoros & $711(22.2)$ & 2,487 (77.8) & 3,198 \\
\hline Ethiopia & 7,997 (72.5) & $3,026(27.5)$ & 11,023 \\
\hline Kenya & 7,308 (37.5) & $12,166(62.5)$ & 19,474 \\
\hline Malawi & $1,227(7.1)$ & $16,157(92.9)$ & 17,384 \\
\hline Mozambique & $317(2.8)$ & 11,195 (97.2) & 11,512 \\
\hline Rwanda & $630(7.9)$ & $7,372(92.1)$ & 8,002 \\
\hline Tanzania & $3,485(34.7)$ & $6,567(65.3)$ & 10,052 \\
\hline Uganda & $3,852(25.2)$ & $11,418(74.8)$ & 15,270 \\
\hline Zambia & $1,482(15.1)$ & $8,359(84.9)$ & 9,841 \\
\hline Zimbabwe & $1,293(20.1)$ & $5,126(79.9)$ & 6,419 \\
\hline \multicolumn{4}{|l|}{ Age } \\
\hline $15-19$ & $1,226(17.3)$ & $5,864(82.7)$ & 7,090 \\
\hline $20-24$ & $6,108(20.2)$ & $24,148(79.8)$ & 30,256 \\
\hline $25-29$ & $8,057(23.8)$ & $25,823(76.2)$ & 33,880 \\
\hline $30-34$ & $6,438(24.3)$ & $20,082(75.7)$ & 26,520 \\
\hline $35-39$ & $4,852(27.5)$ & $12,833(72.5)$ & 17,685 \\
\hline $40-44$ & $2,450(30.5)$ & $5,573(69.5)$ & 8,023 \\
\hline $45-49$ & $792(34.0)$ & $1,540(66.0)$ & 2,332 \\
\hline \multicolumn{4}{|l|}{ Marital status } \\
\hline Currently married & 26,355 (24.5\%) & $81,008(75.5 \%)$ & 107,363 \\
\hline Never married & $824(13.4 \%)$ & $5,316(86.6 \%)$ & 6,140 \\
\hline Formerly/ever married & $2,744(22.3 \%)$ & $9,539(77.7 \%)$ & 12,283 \\
\hline
\end{tabular}




\begin{tabular}{|c|c|c|c|}
\hline \multirow[t]{2}{*}{ Variables } & \multicolumn{2}{|l|}{ Home delivery } & \multirow[t]{2}{*}{ Total } \\
\hline & Yes (\%) & No $(\%)$ & \\
\hline Uneducated & $11,914(39.6 \%)$ & $18,181(60.4 \%)$ & 30,095 \\
\hline Primary & $15,274(22.9 \%)$ & $51,379(77.1 \%)$ & 66,653 \\
\hline Secondary & $2,583(10.5 \%)$ & $21,930(89.5 \%)$ & 24,513 \\
\hline Higher & $149(3.3 \%)$ & $4,366(96.7 \%)$ & 4,515 \\
\hline Don't know & $3(30.0 \%)$ & $7(70.0 \%)$ & 10 \\
\hline \multicolumn{4}{|c|}{ Place of residence } \\
\hline Urban & $2,842(9.9 \%)$ & $25,812(90.1 \%)$ & 28,654 \\
\hline Rural & $27,081(27.9 \%)$ & $70,051(72.1 \%)$ & 97,132 \\
\hline \multicolumn{4}{|l|}{ Wealth index } \\
\hline Poorest & $10,801(36.2 \%)$ & $19,051(63.8 \%)$ & 29,852 \\
\hline Poorer & $7,863(29.1 \%)$ & $19,114(70.9 \%)$ & 26,977 \\
\hline Middle & $5,884(24.3 \%)$ & $18,315(75.7 \%)$ & 24,199 \\
\hline Richer & $3,974(17.1 \%)$ & $19,309(82.9 \%)$ & 23,283 \\
\hline Richest & $1,401(6.5 \%)$ & $20,074(93.5 \%)$ & 21,475 \\
\hline \multicolumn{4}{|c|}{ Sex of head of household } \\
\hline Male & $23,558(24.5 \%)$ & $72,563(75.5 \%)$ & 96,121 \\
\hline Female & $6,365(21.5 \%)$ & $23,300(78.5 \%)$ & 29,665 \\
\hline \multicolumn{4}{|c|}{ Age of head of household } \\
\hline $15-19$ & $90(14.0 \%)$ & $553(86.0 \%)$ & 643 \\
\hline $20-24$ & $1,676(18.2 \%)$ & $7,541(81.8 \%)$ & 9,217 \\
\hline $25-29$ & $4,716(20.6 \%)$ & $18,144(79.4 \%)$ & 22,860 \\
\hline $30-34$ & $5,921(22.5 \%)$ & $20,373(77.5 \%)$ & 26,294 \\
\hline $35-39$ & $5,782(25.5 \%)$ & $16,935(74.5 \%)$ & 22,717 \\
\hline $40-44$ & $4,191(27.5 \%)$ & $11,043(72.5 \%)$ & 15,234 \\
\hline $45-49$ & 2,549 (26.9\%) & $6,928(73.1 \%)$ & 9,477 \\
\hline$>49$ & $4,998(25.8 \%)$ & $14,346(74.2 \%)$ & 19,344 \\
\hline
\end{tabular}




\begin{tabular}{|llll|}
\hline Variables & \multicolumn{2}{l}{ Home delivery } & \multirow{2}{*}{ Total } \\
\cline { 2 - 3 } & Yes (\%) & No (\%) & \\
\hline No & $14,151(32.5 \%)$ & $29,330(67.5 \%)$ & 43,481 \\
\hline Yes & $15,758(19.2 \%)$ & $66,472(80.8 \%)$ & 82,230 \\
\hline Total children ever born & & & \\
\hline $1-3$ & $11,591(16.9 \%)$ & $56,906(83.1 \%)$ & 68,497 \\
\hline $4-6$ & $11,458(28.9 \%)$ & $28,113(71.1 \%)$ & 39,571 \\
\hline $7-9$ & $5,583(38.0 \%)$ & $9,115(62.0 \%)$ & 14,698 \\
\hline$>9$ & $1,291(42.7 \%)$ & $1,729(57.3 \%)$ & 3,020 \\
\hline
\end{tabular}

\section{Factors associated with home delivery}

From fitted four models (null model, individual level, cluster level and both cluster and individual level factors), model with both cluster and individual level factors was found optimal model (variance $=1.33, p$ $<0.001$ ). Accordingly, respondent's age group, marital status, educational status, place of residence, living country, wealth index, media exposure and total children ever born were shown significantly associated with the home delivery in the East Africa counties.

In this final best fit model, about $29 \%$ of the variability among communities in the odds of a home delivery was due to the community-level factors (ICC $=28.90 \%$ ) and about $24 \%$ of the variance in the odds of home delivery (PCV $=24.0 \%$ ) across clusters was attributed to both individual and community-level factors. The MOR (4.96) showed that the unexplained heterogeneity between clusters (EAs) was of greater relevance than the individual variables considered in the analysis for understanding the pattern of home delivery.

The odds of home delivery were $16 \%$ times lower among both mothers younger than 20 years (AOR = $0.84,95 \% \mathrm{Cl}: 0.77,0.92)$ and mothers older than 34 years (AOR $=0.84,95 \% \mathrm{Cl}: 0.79,0.89)$ compared to women age group 20-34. Never married and formerly married women had $22 \%$ (AOR $=1.22,95 \% \mathrm{Cl}: 1.11$, $1.35)$ and $31 \%(A O R=1.31,95 \% \mathrm{Cl}: 1.22,1.39)$ more likely to delivery at home as compared to currently married women. Those women at primary educational level, secondary level and higher (tertiary) level had $30 \%(A O R=0.70,95 \% \mathrm{Cl}: 0.67,0.74), 60 \%(\mathrm{AOR}=0.40,95 \% \mathrm{Cl}: 0.37,0.43)$ and $86 \%(\mathrm{AOR}=0.14,95 \%$ Cl: $0.11,0.17)$ less likely to deliver at home as compared to uneducated women. The likelihood of home delivery was 2.27 ( $\mathrm{AOR}=2.28,95 \% \mathrm{Cl}: 2.07,2.49)$ times higher among women living in rural areas in compared with urban women.

The likelihood of home delivery was 2.24 times ( $\mathrm{AOR}=2.24,95 \% \mathrm{Cl}: 1.96,2.56)$ higher among Ethiopian women than Kenyan women. On the other hand, odds of home delivery was decreased by $98 \%$ (AOR = 
$0.02,95 \% \mathrm{Cl}: 0.02,0.03)$ among women from Mozambique, $96 \%(\mathrm{AOR}=0.04,95 \% \mathrm{Cl}: 0.04,0.05)$ Malawian women, $94 \%(\mathrm{AOR}=0.06,95 \% \mathrm{Cl}$ : 0.05, 0.07) Rwandan women, 92\% (AOR $=0.08,95 \% \mathrm{Cl}: 0.07$, $0.09)$ Burundian women, $85 \%(\mathrm{AOR}=0.15,95 \% \mathrm{Cl}$ : $0.13,0.18]$ Zambian women, $75 \%(\mathrm{AOR}=0.25,95 \mathrm{Cl}$ : $0.20,0.31$ ) women from Comoros, $69 \%$ ( $\mathrm{AOR}=0.31,95 \% \mathrm{Cl}$ : $0.27,0.37$ ) Zimbabwean women, $66 \%$ (AOR $=0.34,95 \% \mathrm{Cl}: 0.30,0.38)$ Ugandan women and 53\% (AOR $=0.47,95 \% \mathrm{Cl} 0.41,0.54)$ Tanzania women in compared with Kenyan women.

In compared with women with poorest wealth status, the odds of home delivery was decreased by $26 \%$ $(\mathrm{AOR}=0.74,95 \% \mathrm{Cl}: 0.71,0.78), 40 \%(\mathrm{AOR}=0.61,95 \% \mathrm{Cl}: 0.57,0.64), 55 \%(\mathrm{AOR}=0.45,95 \% \mathrm{Cl}: 0.42,0.48)$ and $73 \%(\mathrm{AOR}=0.27,95 \% \mathrm{Cl}: 0.24,0.29)$ among women with poorer, middle, richer and richest wealth status respectively. Those women who exposed to media were $16 \%$ (AOR $=0.84,95 \% \mathrm{Cl}: 0.81,0.88)$ lower chances of home delivery in compared with women without media exposure. The likelihood of home delivery was increased by $16 \%(\mathrm{AOR}=1.16,95 \% \mathrm{Cl}: 1.15,1.18)$ as a total number of children was increased by one child. The likelihood of home delivery was decreased by $8 \%(A O R=0.92,95 \% \mathrm{Cl}$ : 0.88 , 0.98) among mothers from household headed by female compared to mothers whose household's head is male (Table 3 ). 
Table 3

Bivariable and multivariable mixed effect GLM analysis of home delivery among women in East Africa countries, 2021.

\begin{tabular}{|c|c|c|c|c|c|}
\hline \multirow[t]{2}{*}{ Variables } & \multicolumn{2}{|c|}{ Home delivery } & \multicolumn{2}{|c|}{ Odds Ratio [95\% Cl] } & \multirow{2}{*}{$\begin{array}{l}\mathrm{P} \text { - } \\
\text { value }\end{array}$} \\
\hline & Yes & No & COR & AOR & \\
\hline \multicolumn{6}{|l|}{ Age in years } \\
\hline Middle (20-34) & 21,653 & 68,716 & 1 & 1 & \\
\hline Early (15-19) & 1,284 & 5,832 & $\begin{array}{l}0.61[0.56 \\
0.66]\end{array}$ & $\begin{array}{l}0.84[0.77, \\
0.92]\end{array}$ & $\begin{array}{l}<.001 \\
0.00\end{array}$ \\
\hline Late (35-49) & 8,390 & 19,821 & $\begin{array}{l}1.50[1.44, \\
1.56]\end{array}$ & $\begin{array}{l}0.84[0.79 \\
0.89]\end{array}$ & $\begin{array}{l}< \\
0.001\end{array}$ \\
\hline \multicolumn{6}{|l|}{ Marital status } \\
\hline Currently married & 26,355 & 81,008 & 1 & 1 & 1 \\
\hline Never married & 824 & 5,316 & $\begin{array}{l}0.68[0.62, \\
0.75]\end{array}$ & $\begin{array}{l}1.22 \text { [1.11, } \\
1.35]\end{array}$ & 0.017 \\
\hline Formerly married & 2,744 & 9,539 & $\begin{array}{l}1.28[1.21 \\
1.36]\end{array}$ & $\begin{array}{l}1.31[1.22, \\
1.39]\end{array}$ & $\begin{array}{l}< \\
0.001\end{array}$ \\
\hline \multicolumn{6}{|l|}{ Educational level } \\
\hline Uneducated & 11,914 & 18,181 & 1 & 1 & \\
\hline Primary & 15,274 & 51,379 & $\begin{array}{l}0.54[0.51 \\
0.56]\end{array}$ & $\begin{array}{l}0.70[0.67, \\
0.74]\end{array}$ & $\dot{0} 001$ \\
\hline Secondary & 2,583 & 21,930 & $\begin{array}{l}0.21[0.20 \\
0.23]\end{array}$ & $\begin{array}{l}0.40[0.37, \\
0.43]\end{array}$ & $\hat{0}_{0.001}$ \\
\hline Higher & 149 & 4,366 & $\begin{array}{l}0.05[0.04, \\
0.07]\end{array}$ & $\begin{array}{l}0.14[0.11, \\
0.17]\end{array}$ & $\begin{array}{l}<.001 \\
0.00\end{array}$ \\
\hline Don't know & 3 & 7 & $\begin{array}{l}0.30[0.05 \\
1.78]\end{array}$ & $\begin{array}{l}0.30[0.05, \\
1.93]^{[}\end{array}$ & 0.206 \\
\hline \multicolumn{6}{|l|}{ Place of residence } \\
\hline Urban & 2,842 & 25,812 & 1 & 1 & \\
\hline Rural & 27,081 & 70,051 & $\begin{array}{l}5.57[4.95, \\
6.28]\end{array}$ & $\begin{array}{l}2.27[2.07, \\
2.49]\end{array}$ & $\begin{array}{l}<.001 \\
0.00\end{array}$ \\
\hline \multicolumn{6}{|l|}{ Country } \\
\hline Kenya & 7,308 & 12,166 & 1 & 1 & \\
\hline Burundi & 1,621 & 11,990 & $\begin{array}{l}0.14[0.12, \\
0.17]\end{array}$ & $\begin{array}{l}0.08[0.07 \\
0.09]\end{array}$ & $\hat{0}_{0.001}$ \\
\hline
\end{tabular}




\begin{tabular}{|c|c|c|c|c|c|}
\hline \multirow[t]{2}{*}{ Variables } & \multicolumn{2}{|c|}{ Home delivery } & \multicolumn{2}{|c|}{ Odds Ratio [95\% Cl] } & \multirow{2}{*}{$\begin{array}{l}\mathrm{P} \text { - } \\
\text { value }\end{array}$} \\
\hline & Yes & No & COR & AOR & \\
\hline Comoros & 711 & 2,487 & $\begin{array}{l}0.29 \text { [0.22, } \\
0.37]\end{array}$ & $\begin{array}{l}0.25[0.20 \\
0.31]\end{array}$ & $\begin{array}{l}< \\
0.001\end{array}$ \\
\hline Ethiopia & 7,997 & 3,026 & $\begin{array}{l}3.20[2.72, \\
3.76]\end{array}$ & $\begin{array}{l}2.24[1.96, \\
2.56]\end{array}$ & $\begin{array}{l}<.001 \\
0.001\end{array}$ \\
\hline Malawi & 1,227 & 16,157 & $\begin{array}{l}0.07[0.05, \\
0.07]\end{array}$ & $\begin{array}{l}0.04[0.04, \\
0.05]\end{array}$ & $\begin{array}{l}<.001 \\
0.00\end{array}$ \\
\hline Mozambique & 317 & 11,195 & $\begin{array}{l}0.02[0.02 \\
0.03]\end{array}$ & $\begin{array}{l}0.02[0.02, \\
0.03]\end{array}$ & $\dot{0} 001$ \\
\hline Rwanda & 630 & 7,372 & $\begin{array}{l}0.07[0.06, \\
0.09]\end{array}$ & $\begin{array}{l}0.06[0.05, \\
0.07]\end{array}$ & $\begin{array}{l}<.001 \\
0.00\end{array}$ \\
\hline Tanzania & 3,485 & 6,567 & $\begin{array}{l}0.52[0.44, \\
0.62]\end{array}$ & $\begin{array}{l}0.47[0.41, \\
0.54]\end{array}$ & $\begin{array}{l}< \\
0.001\end{array}$ \\
\hline Uganda & 3,852 & 11,418 & $\begin{array}{l}0.44[0.37 \\
0.51]\end{array}$ & $\begin{array}{l}0.34[0.30, \\
0.38]\end{array}$ & $\begin{array}{l}<.001 \\
0.001\end{array}$ \\
\hline Zambia & 1,482 & 8,359 & $\begin{array}{l}0.18[0.15, \\
0.21]\end{array}$ & $\begin{array}{l}0.15[0.13, \\
0.18]\end{array}$ & $\begin{array}{l}<.001 \\
0.00\end{array}$ \\
\hline Zimbabwe & 1,293 & 5,126 & $\begin{array}{l}0.20[0.16, \\
0.24]\end{array}$ & $\begin{array}{l}0.31[0.27, \\
0.37]\end{array}$ & $\begin{array}{l}< \\
0.001\end{array}$ \\
\hline \multicolumn{6}{|l|}{ Wealth index } \\
\hline Poorest & 10,801 & 19,051 & 1 & 1 & \\
\hline Poorer & 7,863 & 19,114 & $\begin{array}{l}0.68 \text { [0.64, } \\
0.71]\end{array}$ & $\begin{array}{l}0.74[0.71 \\
0.78]\end{array}$ & $\begin{array}{l}< \\
0.001\end{array}$ \\
\hline Middle & 5,884 & 18,315 & $\begin{array}{l}0.50[0.48, \\
0.53]\end{array}$ & $\begin{array}{l}0.60[0.57, \\
0.64]\end{array}$ & $\begin{array}{l}< \\
0.001\end{array}$ \\
\hline Richer & 3,974 & 19,309 & $\begin{array}{l}0.32[0.30 \\
0.34]\end{array}$ & $\begin{array}{l}0.45[0.42, \\
0.48]\end{array}$ & $\begin{array}{l}<.001 \\
0.001\end{array}$ \\
\hline Richest & 1,401 & 20,074 & $\begin{array}{l}0.13[0.12 \\
0.14]\end{array}$ & $\begin{array}{l}0.27[0.24 \\
0.29]\end{array}$ & $\dot{L}_{0.001}$ \\
\hline \multicolumn{6}{|l|}{$\begin{array}{l}\text { Sex of head of } \\
\text { household }\end{array}$} \\
\hline Male & 23,558 & 72,563 & 1 & 1 & \\
\hline Female & 6,365 & 23,300 & $\begin{array}{l}1.01[0.97, \\
1.06]\end{array}$ & $\begin{array}{l}0.92[0.88, \\
0.98]\end{array}$ & 0.003 \\
\hline
\end{tabular}




\begin{tabular}{|c|c|c|c|c|c|}
\hline \multirow[t]{2}{*}{ Variables } & \multicolumn{2}{|c|}{ Home delivery } & \multicolumn{2}{|c|}{ Odds Ratio [95\% Cl] } & \multirow{2}{*}{$\begin{array}{l}\mathrm{P}- \\
\text { value }\end{array}$} \\
\hline & Yes & No & COR & AOR & \\
\hline $15-19$ & 90 & 553 & 1 & 1 & \\
\hline $20-24$ & 1,676 & 7,541 & $\begin{array}{l}1.39[1.04 \\
1.85]\end{array}$ & $\begin{array}{l}1.21 \text { [0.91, } \\
1.61]\end{array}$ & 0.199 \\
\hline $25-29$ & 4,716 & 18,144 & $\begin{array}{l}1.60[1.21 \\
2.12]\end{array}$ & $\begin{array}{l}1.25[0.94 \\
1.66]\end{array}$ & 0.127 \\
\hline $30-34$ & 5,921 & 20,373 & $\begin{array}{l}1.88[1.42 \\
2.48]\end{array}$ & $\begin{array}{l}1.25[0.94, \\
1.67]\end{array}$ & 0.126 \\
\hline $35-39$ & 5,782 & 16,935 & $\begin{array}{l}2.19[1.66, \\
2.90]\end{array}$ & $\begin{array}{l}1.28[0.96 \\
1.71]\end{array}$ & 0.091 \\
\hline $40-44$ & 4,191 & 11,043 & $\begin{array}{l}2.25[1.70 \\
2.98]\end{array}$ & $\begin{array}{l}1.18[0.88, \\
1.57]\end{array}$ & 0.27 \\
\hline $45-49$ & 2,549 & 6,928 & $\begin{array}{l}2.19 \text { [1.65, } \\
2.92]\end{array}$ & $\begin{array}{l}1.13[0.84, \\
1.51]\end{array}$ & 0.423 \\
\hline$>49$ & 4,998 & 14,346 & $\begin{array}{l}1.86[1.41, \\
2.47]\end{array}$ & $\begin{array}{l}1.18[0.89, \\
1.57]\end{array}$ & 0.258 \\
\hline \multicolumn{6}{|l|}{ Media exposure } \\
\hline No & 14,151 & 29,330 & 1 & 1 & \\
\hline Yes & 15,758 & 66,472 & $\begin{array}{l}0.59 \text { [0.57, } \\
0.62]\end{array}$ & $\begin{array}{l}0.84[0.81 \\
0.88]\end{array}$ & $\begin{array}{l}< \\
0.001\end{array}$ \\
\hline $\begin{array}{l}\text { Total children } \\
\text { ever born (mean } \pm \text { SD) }\end{array}$ & $\begin{array}{l}4.66[ \pm \\
2.52]\end{array}$ & $\begin{array}{l}3.52[ \pm \\
2.26]\end{array}$ & $\begin{array}{l}1.18[1.18, \\
1.19]\end{array}$ & $\begin{array}{l}1.16[1.15, \\
1.18]\end{array}$ & $\begin{array}{l}< \\
0.001\end{array}$ \\
\hline Variance & & & & $\begin{array}{l}1.33 \text { [1.26, } \\
1.42]\end{array}$ & \\
\hline PCV (\%) & & & & 24.0 & \\
\hline ICC (\%) & & & & 28.90 & \\
\hline MOR & & & & 4.96 & \\
\hline
\end{tabular}

\section{Discussion}

This study included a total of 125,696 women who gave birth in the 5 years preceding each resent survey conducted in East African Countries. The prevalence of Home delivery was wide-ranging among countries in Easter Africa (ranged from 2.85\% in Mozambique to $72.5 \%$ in Ethiopia). The prevalence of home 
delivery was associated with respondent's age group, marital status, educational status, place of residence, living Country, wealth index, media exposure and total children ever born.

The pooled prevalence of home delivery from East African Region is consistent with report of India DHS report (22\%)[17], and lower than studies conducted in Nigeria [18]. When compared to the Kenyan, the likelihood of home delivery was 2.24 times higher among Ethiopian. But the likelihood of home delivery was decreased by $98 \%$ for Mozambique, $96 \%$ for Malawian, $94 \%$ for Rwandan, 92 for Burundian, $85 \%$ for Zambian, $75 \%$ for Comoros, $69 \%$ for Zimbabwean, $66 \%$ for Ugandan. The geographical locations of studies varied widely with populations with differing background characteristics and social customs. In addition to social determinants, the health service coverage, quality of maternal healthcare services, economical and health policy of the country might have a role in reducing the home delivery [19].

The likelihood of home delivery was lowered by $16 \%$ among women aged 15-19 years and 35-49 years compared to middle age group women (20-35 years). This association is similar with the result of previous study [2]. Qualitative study conducted in Zambia suggested young women and those without experience in childbirth consulted their parents for the place of delivery. Other studies also reported home preference to give birth is more likely among multiparous mothers. This might they believe that they should not be delivered by either a young nurse or a male staff at the clinic [20]. Cross sectional study conducted in Ethiopia [21] also suggested preference for a health facility delivery was largely due to the understanding that if complications occurred and this was the only place where they could be managed. Hence self-perception, women attitude and fear of complication might be the factors reduce home delivery during the early (young age) and late (advanced age) pregnancies.

Currently unmarried (never married and formerly married) women were more likely to delivery at home as compared to currently married women. Theories linking marital status, pregnancy and birth preparedness indicated that unmarried women faced lack or reduced level of psychosocial support and relationship stability [22]. Pregnant women without marriage might be unplanned and/ or unwanted. On other hand, low social acceptance of unmarried status in that there is still social stigmatism surrounding illegitimate births in many countries. Hence unmarried women may be intrinsically different from married women who may be less empowered, self-isolated or lack of motivation to access the health service [23-25]. All these factors might be increasing the likelihood of home delivery among currently unmarried mothers.

Educated women had less likelihood of home deliver as compared to uneducated women. The result was in line with the individual studies conducted in rural Ethiopia [26-28], Zimbabwe [29], Nigeria [30], Ghana [31, 32], Guinea [23] and Nepal [33]. The reason for this might be due the fact that when mothers are educated it is more is likely to enhance female self-determination, positive attitude, and financial freedom $[34,35]$. Furthermore, it is more likely that educated women demand higher quality service and be alert of difficulties during pregnancy as well as child birth. As a result, they are more probable to use maternal health care services unlike that the illiterate one [36]. These could collectively influence mothers' awareness to seek better maternal health services, including delivering in health facilities. 
Women living rural area had as higher odds of home delivery, which is similar to findings in previous studies [23, 28, 31, 37]. Rural residents in Sub Saharan countries have poor access to health care facilities. Moreover, lack of privacy and confidentiality, and negligence in the provision of care during childbirth by skilled birth attendants are the fear of the women [38]. With rural healthcare provider shortages, greater travel distances and very limited access to obstetric care, it could be likely that there would be high risk of home delivery in rural areas.

In compared with women with poorest wealth status, the odds of home delivery were decreased by $26 \%$, $40 \%, 55 \%$ and $73 \%$ among women with poorer, middle, richer and richest wealth status, respectively. This result was in agreement with the previous studies [26-28,31]. Financial capability of families and costs related to transportation may determine the place of delivery. Moreover, women from higher wealth status might be more empowered, participate in decision and seek maternal health service.

Consistent with previous studies $[23,28,39,40]$ our finding suggests that women who exposed to media were $20 \%$ lower chances of home delivery in compared with women without media exposure. Media promotion of institutional delivery and danger of home delivery may influence mothers to develop positive behavior towards delivering in a health facility.

The other most significant determinants of home delivery this study was number of children. The likelihood of home delivery was increased by $16 \%$ as the number of total children in the household is increased by one child. This finding was in line with previous studies conducted in Ethiopia [23, 28]. Since the women normalize childbirth, they might be less likely to seek care during labor [41]. Literature also indicated women's previous interactions with health facilities have an influence and poor experiences during past deliveries may discourage women from returning for the next birth [42, 43]. Hence, the less fear of complication, adverse experience of care for women during childbirth could discourage them to use health facility services in subsequent pregnancies. In addition to these, Multiparous mothers who had done their previous deliveries at home might be more likely to deliver at home in their recent pregnancy $[44,45]$.

This further analysis of data obtained from nationally representative in the East Africa DHS dataset was population-based with high response rate. The sample size used is large enough to detect the association factors with high power of the study. Hence this study is beneficial to inform policymakers and planners for their intervention to line up.

Limitations of the study include as in any cross-sectional nature of the study design the finding from this study may not found a true causal association between the home delivery and covariate. The data was collected based on self-report from mothers within 5 years prior the survey and this could be a potential source of recall bias. There was no information on numerous other important factors of maternal health service use during childbirth including the existence of emergencies during home deliveries that prompt seeking professional assistance and outcomes from past healthcare service use. Additionally, some important factors like antenatal care, obstetric histories are not included in the analysis as there was no similar definition or classification among the included countries. Since some countries have no DHS 
program, some countries have limited data access, and some excluded due to old survey (more than ten

years) the result of this study may not representative of the entire East Africa zone.

\section{Conclusion}

Home delivery was varied between countries of East African zone. The home delivery was significantly increased among women with middle aged, high number of ever born children, rural residence, never married or formerly married participants. To the contrast home delivery was decreased with higher educational level, media exposure, and higher wealth index.

Wide range interventions to decrease home delivery should focus on addressing inequities associated with maternal education, family wealth, increased access to media, as well as narrowing the gap between the rural and the urban areas, poor and rich families, and married and unmarried mothers.

\section{Abbreviations}

AOR: Adjusted Odds Ratio; Cl: Confidence Interval; CSA: Central Statistics Agency; DHS: Demography heath survey; EAs: Enumeration Areas; GLMM: Generalized Linear Mixed Models; ICC: Intra-cluster Correlation Coefficient; IR: Individual Record; LMIC: Low and Middle-Income Countries; LR: Likelihood Ratio; MMR: Maternal Mortality Ratio; PCV: Proportional Change in Variance; PPH: Postpartum Hemorrhage; SSA: Sub-Saharan African (SSA); WHO; World Health Organization;

\section{Declarations}

\section{Acknowledgments}

We would like to express our very profound gratitude to the measure DHS for providing us the data and shape files for the study area.

\section{Ethics approval and consent to participate}

We requested DHS Program, and permission was granted by 149848 reference number to download and use the data for this study from http://www.dhsprogram.com. There are no individual identifiers reported in any part of this manuscript. All the data management and analysis were strictly followed the standard indicated in the manuals of DHS.

\section{Consent for publication}

Not applicable.

\section{Availability of data and materials}

All necessary information's were included with the manuscript. 


\section{Competing interest}

All authors declare no conflicts of interest.

\section{Funding}

No fund was received for this work.

\section{Authors' contributions}

Conception of the work, design of the work, acquisition of data, analysis, and interpretation of data were conducted by LD and BS. Data curation, drafting the article, revising it critically for intellectual content, validation, and final approval of the version to be published were done by LD, AT, AB and BS. All authors read and approved the final manuscript.

\section{References}

1. WHO U. UNFPA, The World Bank. Trends in maternal mortality: 1990 to 2010. World Health Organization, UNICEF, UNFPA, and The World Bank. 2012.

2. Mrisho M, Schellenberg JA, Mushi AK, Obrist B, Mshinda H, Tanner M, et al. Factors affecting home delivery in rural Tanzania. Tropical medicine \& international health. 2007;12(7):862-72.

3. WHO U. UNFPA, World Bank Group and the United Nations Population Division. Trends in maternal mortality: 1990 to 2015: estimates by WHO, UNICEF. UNFPA, World Bank group and the united nations population division; 2015.

4. Who U. UNFPA, World bank. Trends in maternal mortality. 1990;2008(2010):1.

5. Comissions UNE. Assessing progress in Africa towards the millennium development goals. 2013.

6. nations U. The millennium development goals report in Time Glob. Action People Planet. 2016.

7. Health FMO. HSTP I Annual Performance Report EFY 2008 (2015/2016). 2017.

8. WHO. Core health indicators. Geneva: World Health Organization Statistical Information System. 2007.

9. Belemsaga DY, Goujon A, Kiendrebeogo JA, Duysburgh E, Kouanda S, Degomme O, et al. A review of factors associated with the utilization of healthcare services and strategies for improving postpartum care in Africa. Afrika Focus. 2015;28(2):83.

10. Say L, Chou D, Gemmill A, Tunçalp Ö, Moller A-B, Daniels J, et al. Global causes of maternal death: a WHO systematic analysis. The Lancet Global Health. 2014;2(6):e323-e33.

11. Callister LC, Edwards JE. Sustainable Development Goals and the ongoing process of reducing maternal mortality. Journal of Obstetric, Gynecologic \& Neonatal Nursing. 2017;46(3):e56-e64.

12. Bank W. New country classifications by income level: 2019-2020 2020 [January 11, 2021]. Available from: https://datahelpdesk.worldbank.org/knowledgebase/articles/906519-world-bank-country-andlending-groups. 
13. Pittsburgh Uo. African Studies and African Country Resources @ Pitt: East African Countries 2021 [updated Jan 8, 2021 11:03 AM; cited 2021 January 11, 2021]. Available from: https://pitt.libguides.com/c.php?g=12378\&p=65814.

14. Agency CS, ICF. Ethiopia Demographic and Health Survey 2016: Key Indicators Report. Addis Ababa, Ethiopia, and Rockville, Maryland, USA. CSA and ICF. 2016.

15. Snijders TAB, Bosker RJ. Multilevel analysis: An introduction to basic and advanced multilevel modeling: Sage; 2011.

16. Merlo J, Chaix B, Ohlsson H, Beckman A, Johnell K, Hjerpe P, et al. A brief conceptual tutorial of multilevel analysis in social epidemiology: using measures of clustering in multilevel logistic regression to investigate contextual phenomena. J Epidemiol Community Health. 2006;60(4):290-7. Epub 2006/03/16. doi: 10.1136/jech.2004.029454. PubMed PMID: 16537344; PubMed Central PMCID: PMCPMC2566165.

17. Ou C-Y, Yasmin M, Ussatayeva G, Lee M-S, Dalal K. Maternal Delivery at Home: Issues in India. Advances in Therapy. 2021;38(1):386-98. doi: 10.1007/s12325-020-01551-3.

18. Ayamolowo LB, Odetola TD, Ayamolowo SJ. Determinants of choice of birth place among women in rural communities of southwestern Nigeria. International Journal of Africa Nursing Sciences. 2020;13:100244. doi: https://doi.org/10.1016/j.ijans.2020.100244.

19. Joseph KJV, Mozumdar A, Lhungdim H, Acharya R. Quality of care in sterilization services at the public health facilities in India: A multilevel analysis. PLoS One. 2020;15(11):e0241499. Epub 2020/11/03. doi: 10.1371/journal.pone.0241499. PubMed PMID: 33137153; PubMed Central PMCID: PMCPMC7605679.

20. Ahmed M, Demissie M, Worku A, Abrha A, Berhane Y. Socio-cultural factors favoring home delivery in Afar pastoral community, northeast Ethiopia: A Qualitative Study. Reprod Health. 2019;16(1):171. Epub 2019/11/23. doi: 10.1186/s12978-019-0833-3. PubMed PMID: 31752897; PubMed Central PMCID: PMCPMC6868681.

21. Belay A, Sendo E. Factors determining choice of delivery place among women of child bearing age in Dega Damot District, North West of Ethiopia: a community based cross- sectional study. BMC Pregnancy Childbirth. 2016;16:229. Epub 2016/08/19. doi: 10.1186/s12884-016-1020-y. PubMed PMID: 27535126; PubMed Central PMCID: PMCPMC4988051.

22. Shah P, Zao J, Ali S. Maternal Marital Status and Birth Outcomes: A Systematic Review and MetaAnalyses. Maternal and child health journal. 2011;15:1097-109. doi: 10.1007/s10995-010-0654-z.

23. Ahinkorah BO. Non-utilization of health facility delivery and its correlates among childbearing women: a cross-sectional analysis of the 2018 Guinea demographic and health survey data. BMC Health Serv Res. 2020;20(1):1016. Epub 2020/11/11. doi: 10.1186/s12913-020-05893-0. PubMed PMID: 33167985; PubMed Central PMCID: PMCPMC7650152.

24. Lowe M, Chen DR, Huang SL. Social and Cultural Factors Affecting Maternal Health in Rural Gambia: An Exploratory Qualitative Study. PLoS One. 2016;11(9):e0163653. Epub 2016/09/24. doi: 
10.1371/journal.pone.0163653. PubMed PMID: 27661617; PubMed Central PMCID:

PMCPMC5035064.

25. Nyblade L, Stockton MA, Giger K, Bond V, Ekstrand ML, Lean RM, et al. Stigma in health facilities: why it matters and how we can change it. BMC Med. 2019;17(1):25. Epub 2019/02/16. doi: 10.1186/s12916-019-1256-2. PubMed PMID: 30764806; PubMed Central PMCID: PMCPMC6376713.

26. Chernet AG, Dumga KT, Cherie KT. Home Delivery Practices and Associated Factors in Ethiopia. J Reprod Infertil. 2019;20(2):102-8. Epub 2019/05/07. PubMed PMID: 31058055; PubMed Central PMCID: PMCPMC6486567.

27. Mitiku AA, Dimore AL, Mogas SB. Determinants of Home Delivery among Mothers in Abobo District, Gambella Region, Ethiopia: A Case Control Study. Int J Reprod Med. 2020;2020:8856576. Epub 2021/01/26. doi: 10.1155/2020/8856576. PubMed PMID: 33490230; PubMed Central PMCID: PMCPMC7787860.

28. Yebyo H, Alemayehu M, Kahsay A. Why do women deliver at home? Multilevel modeling of Ethiopian National Demographic and Health Survey data. PLoS One. 2015;10(4):e0124718. Epub 2015/04/16. doi: 10.1371/journal.pone.0124718. PubMed PMID: 25874886; PubMed Central PMCID: PMCPMC4398378.

29. Nunu WN, Ndlovu V, Maviza A, Moyo M, Dube O. Factors associated with home births in a selected ward in Mberengwa District, Zimbabwe. Midwifery. 2019;68:15-22. Epub 2018/10/14. doi: 10.1016/j.midw.2018.09.013. PubMed PMID: 30316175.

30. Ashimi AO, Amole TG. Prevalence, reasons and predictors for home births among pregnant women attending antenatal care in Birnin Kudu, North-west Nigeria. Sex Reprod Healthc. 2015;6(3):119-25. Epub 2016/02/05. doi: 10.1016/j.srhc.2015.01.004. PubMed PMID: 26842633.

31. Ahinkorah BO, Seidu AA, Budu E, Agbaglo E, Appiah F, Adu C, et al. What influences home delivery among women who live in urban areas? Analysis of 2014 Ghana Demographic and Health Survey data. PLoS One. 2021;16(1):e0244811. Epub 2021/01/05. doi: 10.1371/journal.pone.0244811. PubMed PMID: 33395424; PubMed Central PMCID: PMCPMC7781474.

32. Budu E. Predictors of home births among rural women in Ghana: analysis of data from the 2014 Ghana Demographic and Health Survey. BMC Pregnancy Childbirth. 2020;20(1):523. Epub 2020/09/12. doi: 10.1186/s12884-020-03211-4. PubMed PMID: 32912164; PubMed Central PMCID: PMCPMC7488046.

33. Shahabuddin A, De Brouwere V, Adhikari R, Delamou A, Bardají A, Delvaux T. Determinants of institutional delivery among young married women in Nepal: Evidence from the Nepal Demographic and Health Survey, 2011. BMJ Open. 2017;7(4):e012446. Epub 2017/04/15. doi: 10.1136/bmjopen2016-012446. PubMed PMID: 28408543; PubMed Central PMCID: PMCPMC5594213.

34. Bayeh $E$. The role of empowering women and achieving gender equality to the sustainable development of Ethiopia. Pacific Science Review B: Humanities and Social Sciences. 2016;2(1):3742. doi: https://doi.org/10.1016/j.psrb.2016.09.013. 
35. Mahmud S, Shah NM, Becker S. Measurement of Women's Empowerment in Rural Bangladesh. World Development. 2012;40(3):610-9. doi: https://doi.org/10.1016/j.worlddev.2011.08.003.

36. Sado L, Spaho A, Hotchkiss DR. The influence of women's empowerment on maternal health care utilization: Evidence from Albania. Social Science \& Medicine. 2014;114:169-77. doi: https://doi.org/10.1016/j.socscimed.2014.05.047.

37. Kitui J, Lewis S, Davey G. Factors influencing place of delivery for women in Kenya: an analysis of the Kenya demographic and health survey, 2008/2009. BMC Pregnancy and Childbirth. 2013;13(1):40. doi: 10.1186/1471-2393-13-40.

38. Adatara P, Strumpher J, Ricks E. Exploring the reasons why women prefer to give birth at home in rural northern Ghana: a qualitative study. BMC Pregnancy and Childbirth. 2020;20(1):500. doi: 10.1186/s12884-020-03198-y.

39. Ganle JK, Mahama MS, Maya E, Manu A, Torpey K, Adanu R. Understanding factors influencing home delivery in the context of user-fee abolition in Northern Ghana: Evidence from 2014 DHS. Int J Health Plann Manage. 2019;34(2):727-43. Epub 2019/01/19. doi: 10.1002/hpm.2731. PubMed PMID: 30657200.

40. Siyoum M, Astatkie A, Mekonnen S, Bekele G, Taye K, Tenaw Z, et al. Home birth and its determinants among antenatal care-booked women in public hospitals in Wolayta Zone, southern Ethiopia. PLoS One. 2018;13(9):e0203609. Epub 2018/09/08. doi: 10.1371/journal.pone.0203609. PubMed PMID: 30192861; PubMed Central PMCID: PMCPMC6128615.

41. Bishanga DR, Drake M, Kim YM, Mwanamsangu AH, Makuwani AM, Zoungrana J, et al. Factors associated with institutional delivery: Findings from a cross-sectional study in Mara and Kagera regions in Tanzania. PLoS One. 2018;13(12):e0209672. Epub 2018/12/27. doi:

10.1371/journal.pone.0209672. PubMed PMID: 30586467; PubMed Central PMCID: PMCPMC6306247.

42. Sialubanje C, Massar K, Hamer DH, Ruiter RA. Reasons for home delivery and use of traditional birth attendants in rural Zambia: a qualitative study. BMC Pregnancy Childbirth. 2015;15:216. Epub 2015/09/13. doi: 10.1186/s12884-015-0652-7. PubMed PMID: 26361976; PubMed Central PMCID: PMCPMC4567794.

43. Kifle MM, Kesete HF, Gaim HT, Angosom GS, Araya MB. Health facility or home delivery? Factors influencing the choice of delivery place among mothers living in rural communities of Eritrea. Journal of Health, Population and Nutrition. 2018;37(1):22. doi: 10.1186/s41043-018-0153-1.

44. Dhakal PM, Shrestha MM, Baral DM, Pathak SM. Factors Affecting the Place of Delivery among Mothers Residing in Jhorahat VDC, Morang, Nepal. Int J Community Based Nurs Midwifery. 2018;6(1):2-11. Epub 2018/01/19. PubMed PMID: 29344531; PubMed Central PMCID: PMCPMC5747568.

45. Baral YR, Lyons K, Skinner J, van Teijlingen ER. Maternal health services utilisation in Nepal: Progress in the new millennium? Health science journal. 2012;6(4):618. 




Figure 1

Schematic presentation of sampled East Africa countries for the pooled analysis of home delivery.

\section{Home delivery}



Figure 2 
shows the prevalence of home delivery among women in East Africa countries, 2021 